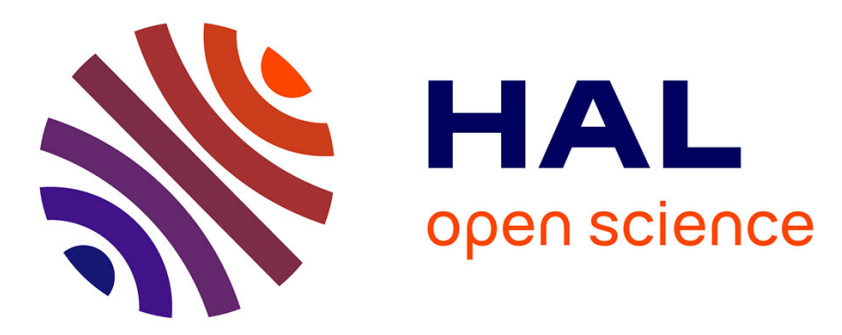

\title{
Near-critical density filling of the SF6 fluid cell for the ALI-R-DECLIC experiment in weightlessness
}

Carole Lecoutre, Samuel Marre, Yves Garrabos, Daniel Beysens, Inseob Hahn

\section{To cite this version:}

Carole Lecoutre, Samuel Marre, Yves Garrabos, Daniel Beysens, Inseob Hahn. Near-critical density filling of the SF6 fluid cell for the ALI-R-DECLIC experiment in weightlessness. Acta Astronautica, 2018, 146, pp.243-248. 10.1016/j.actaastro.2018.03.004 . hal-01764226

\section{HAL Id: hal-01764226 \\ https://hal.science/hal-01764226}

Submitted on 13 Apr 2018

HAL is a multi-disciplinary open access archive for the deposit and dissemination of scientific research documents, whether they are published or not. The documents may come from teaching and research institutions in France or abroad, or from public or private research centers.
L'archive ouverte pluridisciplinaire HAL, est destinée au dépôt et à la diffusion de documents scientifiques de niveau recherche, publiés ou non, émanant des établissements d'enseignement et de recherche français ou étrangers, des laboratoires publics ou privés. 
Near-critical density filling of the $\mathrm{SF}_{6}$ fluid cell for the ALI-R-DECLIC experiment in weightlessness ${ }^{*}$

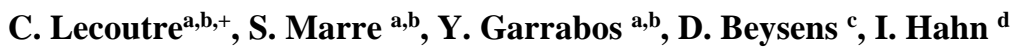 \\ ${ }^{\text {a } C N R S, ~ I C M C B ~ E S E M E, ~ U M R ~ 5026, ~ F-33600 ~ P e s s a c, ~ F r a n c e, ~}$ \\ ${ }^{\mathrm{b}}$ Univ. Bordeaux, ICMCB, UMR 5026, F-33600 Pessac, France \\ c Laboratoire de Physique et Mécanique des Milieux Hétérogènes, CNRS PSL-ESPCI-Sorbonne Paris Cité, 10 \\ rue Vauquelin, F-75005 Paris, France CNRS PSL-ESPCI-Sorbonne Université-Sorbonne Paris Cité, \\ 10 rue Vauquelin, F-75005 Paris, France \\ ' Jet Propulsion Laboratory, California Institute of Technology, California 91109, USA \\ + Corresponding Author at : ICMCB-CNRS, 87 Avenue du Dr Albert Schweitzer, 33600 Pessac, France, \\ carole.lecoutre@icmcb.cnrs.fr
}

\begin{abstract}
Analyses of ground-based experiments on near-critical fluids to precisely determine their density can be hampered by several effects, especially the density stratification of the sample, the liquid wetting behavior at the cell walls, and a possible singular curvature of the "rectilinear" diameter of the density coexisting curve. For the latter effect, theoretical efforts have been made to understand the amplitude and shape of the critical hook of the density diameter, which depart from predictions from the so-called ideal lattice-gas model of the uniaxial 3DIsing universality class. In order to optimize the observation of these subtle effects on the position and shape of the liquid-vapor meniscus in the particular case of $\mathrm{SF}_{6}$, we have designed and filled a cell that is highly symmetrized with respect to any median plane of the total fluid volume. In such a viewed quasi-perfect symmetrical fluid volume, the precise detection of the meniscus position and shape for different orientations of the cell with respect to the Earth's gravity acceleration field becomes a sensitive probe to estimate the cell mean density filling and to test the singular diameter effects. After integration of this cell in the ALI-R insert, we take benefit of the high optical and thermal performances of the DECLIC Engineering Model. Here we present the sensitive imaging method providing the precise ground-based $\mathrm{SF}_{6}$ benchmark data. From these data analysis it is found that the temperature dependence of the meniscus position does not reflect the expected critical hook in the rectilinear density diameter. Therefore the off-density criticality of the cell is accurately estimated, before near future experiments using the same ALI-R insert in the DECLIC facility already on-board the International Space Station.
\end{abstract}

Keywords: slightly off-critical sulfur-hexafluoride, liquid-gas density diameter, liquid-gas coexisting densities

\footnotetext{
* This paper IAC-17-A2,4 was presented at $68^{\text {th }}$ International Astronautical Congress (IAC), 25-29 September 2017, Adelaïde, Australia
}

\section{Introduction}

Thermodynamic and transport properties show singularities asymptotically close to the critical points of many different systems. The current theoretical paradigm on critical phenomena using renormalization group (RG) approach [1] has ordered these systems in well-defined universality classes [2] and has characterized the asymptotic singularities in terms of power laws of only two relevant scaling fields [3] in order to be conform to the scaling hypothesis. Simple fluids are then assumed similar [4] to the $\mathrm{O}(1)$ symmetric $\left(\Phi^{2}\right)^{2}$ field theory and the $\mathrm{N}=1$ vector model of three-dimensional (3D) Ising-like systems ([2], [5]). Their study in weightlessness condition is well-recommended to test the two-scalefactor universality approaching their critical point. However, for the case of the gas-liquid critical point of simple fluids, some additional difficulties can occur as the order parameter - the fluctuating local density shows a noticeable asymmetry, as for instance the well-known rectilinear diameter form of the liquid-gas coexisting density curve first evidenced by Cailletet and Mathias [6]. This linear asymmetry was largely confirmed in the subsequent literature (see for instance Ref. [7]). Such asymmetrical effects cannot be accounted for from the symmetrical uniaxial 3D Ising model and its induced standard fluid-like version, i.e., the symmetrical lattice-gas model.

An alternative theoretical way to introduce the fluid asymmetry nature in the scaling approach consists in extending the number of the physical fields contributing explicitly to the relevant scaling fields, the so-called complete scaling phenomenological hypothesis ([8]-[12]). For example, in a recent work [13], Yang-Yang and singular diameter critical anomalies arise in exactly soluble compressible cell gas models where complete scaling includes pressure mixing. The predictions of complete scaling have 
been tested against experiments and simulations at finite distance from the critical point, increasing the complexity in the fundamental quest of a true asymptotic fluid behavior. The latter remains a conundrum to the scientists who have for objective to check it by performing an experiment closer and closer to the critical point with the required precision. De facto, the asymmetrical contributions, the analytical backgrounds, and the classical-to-critical crossover behavior due to the mean-field-like critical point, further hindered the test of the asymptotic Ising-like fluid behavior. Such difficulties are intrinsically ineludible, even along the true critical paths where the crossover contribution due to one additional non-relevant field [14] can be accounted for correctly in the field theory framework ([15]-[16]).

Moreover, the experiments are never exactly on these critical paths, adding paradoxically a new opportunity to investigate the theoretical expectations related to the non-symmetrical behaviors. Indeed, even though the temperature can be made very close to $T_{c}$, the mean density of the fluid cell is never at its exact critical density value [17]. The error-bar related to this latter critical parameter was never contributing to the discussion of the Earth's based results in terms of true experimental distance to the critical point. Nevertheless, from the above experimental facts and the theoretical expectations, it appears that the related non-symmetrical effects can be unambiguously viewed in a slightly off-critical (liquid-like) cell. Indeed, in such a closed liquid-like cell, it is expected that the meniscus position crosses the median volumetric plane at a single finite temperature distance below the coexistence temperature. From the symmetrical lattice-gas model, we recall that the meniscus of any liquid-like cell is expected to be visible always above this median volumetric plane in the two-phase temperature range.

Therefore, an academic interest to use gravity field acceleration to horizontally stabilize the position of the liquid gas meniscus inside eight different cell positions is precisely investigated during the pre-flight determination of the off-critical mean density of a fluid cell before its use under weightlessness environment. More specifically, we would like to check if $\mathrm{SF}_{6}$ remains well similar - or not - to the 1974 standard $\mathrm{SF}_{6}$ fluid ([18]-[20]) which support fluid asymmetry resulting from complete scaling hypothesis ([10]-[12]). Our experimental challenge is then to detect the previously observed significant hook (of $0.5 \%$ amplitude) in the rectilinear density diameter when the relative uncertainty in the filling density value is controlled with $0.1 \%$ precision along a non-critical path which exceeds from $\sim 0.2 \%$ the exact critical isochore. This experimental challenge is illustrated in Fig. 1.

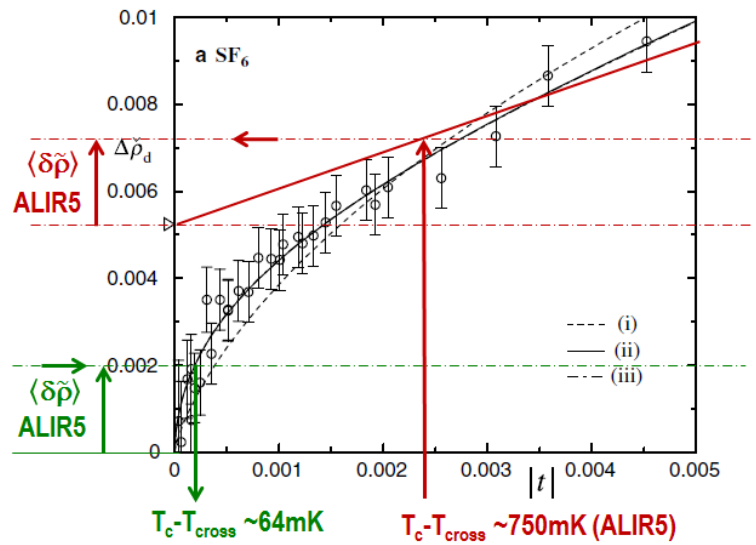

Fig. 1. Experimental critical hook of the linear density diameter of $\mathrm{SF}_{6}$ reported in [10] and related temperature $T_{c}-T_{\text {cross }}$ where the meniscus position must be collapsed on the volumetric median plane of a cylindrical cell filled at the liquid-like off-critical density of $0.20 \%$. Green lines: Expected from the singular hook of the density diameter. Red lines: expected from the rectilinear density diameter

The high optical and thermal performances of the ALI-R insert used in the DECLIC facility Engineering Model allow the observation of the meniscus position behavior precisely due to the density diameter behavior, as the temperature of highly symmetrical test cells is changed. Each of these test cells consists in a quasi-perfect disk-shaped cylindrical fluid volume observed in light transmission, surrounded by two opposite, small, and similar dead volumes. The latter volumes define the single remaining transverse (non-cylindrical) axis of the fluid volume due to the cell in-line filling setup. Here, the selected test cell ALIR5 [21] was filled at a liquid-like mean density $\langle\delta \tilde{\rho}\rangle=\left(\rho / \rho_{c}\right)-1$ very close to the critical density $\rho_{\mathrm{c}}$ of $\mathrm{SF}_{6}$. The relative off-critical density $\langle\delta \tilde{\rho}\rangle=$ $+0.20_{-0.04}^{+0.04} \%$ of ALIR5 was measured with great accuracy from our Earth's-based filling and checking processes (see below $\S 6$ and Ref. [22]). The fluid under study is $\mathrm{SF}_{6}$ of electronic quality, corresponding to $99.995 \%$ purity (from Alpha Gaz - Air Liquide). The meniscus behavior could be analyzed in eight cell configurations. Such analyses provide an accurate experimental evaluation of the relative effects of (i) the complete cell design, (ii) the cell displacement in front of the CCD camera, (iii) the meniscus optical observations through gravitational stratification and liquid wettability, (iv) the cell filling mean density, and finally, (v) the coexisting density diameter behavior. Only the evaluations of (iv) and (v) are treated hereafter.

\section{Experimental set-up and methods}

\subsection{Highly-symmetrical cell design}

The essential characteristic of the ALIR5 cell (see Fig. 2(a)) is its highly symmetrical design with respect to any median plane of the observed cylindrical fluid volume. The main part of the fluid 
sample consists in a fluid layer of thickness $e_{f}=$ $(2.510 \pm 0.002) \mathrm{mm}$ and diameter $d_{f}=2 R=$ $(10.606 \pm 0.005) \mathrm{mm}$. This fluid layer is confined between two flat, parallel, and transparent sapphire windows of thickness $e_{w}=8.995 \mathrm{~mm}$ and external diameter $d_{w}=12 \mathrm{~mm}$. An engraved circle of $10 \mathrm{~mm}$ diameter, $30 \mu \mathrm{m}$ thickness, is deposited on each sapphire external surface. Such a pancake cell design [23] leads to the ALIR5 viewed cylindrical fluid volume of $V_{f v}=\pi R^{2} e_{f}=\left(221.7_{-0.70}^{+0.20}\right) \mathrm{mm}^{3}$ and the ALIR5 cross-sectional area of $A_{f v}=2 R e_{f}=$ $(26.621 \pm 0.020) \mathrm{mm}^{2}$ for any viewed median volumetric plane, except around the direction of the fill-line setup, as detailed below.

The cell body is made of a machined $\mathrm{CuCo}_{2} \mathrm{Be}$ parallelepipedic block of external dimensions $L(=25) \times l(=27) \times h(=24) \mathrm{mm}^{3}$. This body contains two similar fill-line dead volumes - each one in the $\Gamma$-like form of two perpendicularly crossed cylindrical holes - located in the median plane of the fluid thickness. These two $\Gamma$-like volumes are symmetrical from the central optical axis used as the rotation axis of the cell. The resulting windowless fluid volume is $(1 / 2) V_{f b}=(7.0 \pm 0.2) \mathrm{mm}^{3}$ on each side of the observed cylindrical fluid volume. Therefore, $V_{f}=V_{f v}+V_{f b}=\left(235.70_{-1.0}^{+0.5}\right) \mathrm{mm}^{3}$ is the total fluid sample volume. All the above dimensional values are from mechanical measurements performed at $20^{\circ} \mathrm{C}$.

The common axis of the two small opposite cylindrical holes opened in the main cylindrical fluid volume defines the single particular direction of the common median plane. In this latter plane occurs the maximum fluid area $\left(A_{f v}+A_{f b}=A_{f v}+\right.$ $\left.(17.8 \pm 1.0) \mathrm{mm}^{2}\right)$ crossing the complete fluid volume. The horizontal position of this median plane is chosen as the zero angle $\left(\theta=0^{\circ}\right)$ of the cell rotation (or $\theta=180^{\circ}$, equivalently, for the opposite configuration of the cell versus the direction of the gravity vector). From reference to this cell direction, the maximum tilted angle $\theta_{m}$ that overlaps the $\Gamma$-like configuration of the dead volume is $\theta_{m} \gtrsim 28^{\circ}$. The $\pm \theta_{m}$-directions are not equivalent versus the liquid (or gas) gravity positionning inside each dead volume (see below § 6).

The experiment is then performed in four cell directions $\theta=\left\{-23.2^{\circ} ; 0^{\circ} ;+22.9^{\circ} ;+90^{\circ}\right\}$ regarding the above single fill-line positions with respect to two reverse orientations of the earth's gravity vector. That permits to analyze potential systematic errors associated with the cell dead volume.

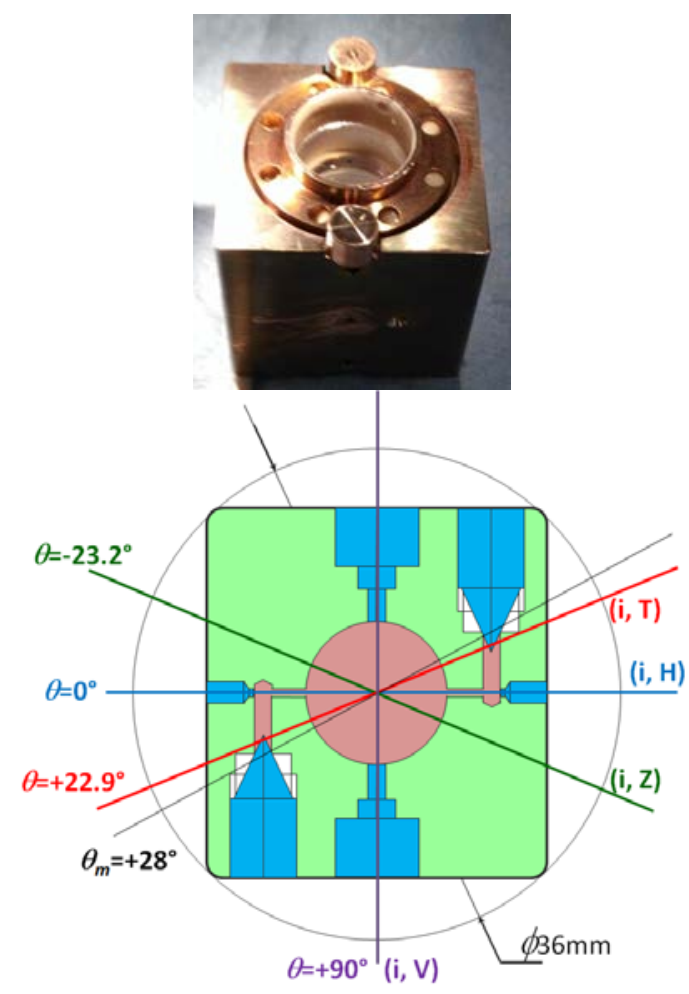

Fig. 2. (a) Picture of the ALIR5 cell. (b) Schematic cross section of the ALIR5 cell where are illustrated the four relative directions of the meniscus $\theta=$ $\left\{-23.2^{\circ} ; 0^{\circ} ;+22.9^{\circ} ;+90^{\circ}\right\}$ and the direction $\theta_{m} \gtrsim 28^{\circ}$ that overlaps the $\Gamma$-like forms of the two symmetrical dead volumes associated to the fill-line direction. Red area: fluid volume; blue area: diffusion windows, filling screws and stoppers; green area: cell body; external circle : dimensional scale .

Throughout the paper, each cell configuration is labeled $\llbracket i, X \rrbracket$ where the digit $i$ represents two reverse gravity orientations ( $\downarrow \downarrow$ for $i=1$ and $\mathrm{g} \uparrow$ for $i=2$ ) and the letter $X$ describes four directions of the fill-line axis of the fluid cell ( $X=H$ for $\theta=0^{\circ}, X=V$ for $\theta=$ $+90^{\circ}, X=T$ for $\theta=22.9^{\circ}$, and $X=Z$ for $\theta=-23.2^{\circ}$ ). The corresponding cross-sectional shape of the cell is schematically pictured in Fig. 2(b), illustrating the relative positions of the meniscus and the dead fluid volumes with respect to the earth's gravity vector.

\subsection{Phase transition temperature.}

The laser light transmission measurements of the EM-DECLIC facility [24] and the wide field-of-view observation of the fluid sample are combined to observe the phase separation process during the cell cooling down. Each temperature quench step crossing the transition temperature (noted $T_{\text {coex }}$ ) is $-1 \mathrm{mK}$. The exact value of $T_{\text {coex }}$ is not essential for the following discussion. The temperature results for each experimental configuration are then reported from reference to the lowest temperature (noted $T_{1 \varphi}$ ) of the monophasic range. The resulting true $\mathrm{SF}_{6}$ coexistence temperature $T_{\text {coex }}$ is such as $0<T_{1 \varphi}-T_{\text {coex }}<1$ $\mathrm{mK}$, noting in addition the high reproducibility $(2 \mathrm{mK}$ range) of $T_{1 \varphi}$ (here from $318.721 \mathrm{~K}$ to $318.723 \mathrm{~K}$ ) for 
the eight experimental runs. Moreover, the $T_{c}-$ $T_{\text {coex }} \simeq 0.4 \mu \mathrm{K}$ shift [25] due to the off-density criticality of the test-cell is neglected, i.e., $T_{c} \sim T_{\text {coex }}$. Finally, wide field-of-view imaging of each meniscus position data is recorded when thermal equilibration is achieved at each temperature difference $\left(T_{1 \varphi}-\right.$ $T) \sim\left(T_{c}-T\right)$. Then $T_{c}-T$ follows a logarithmic-like scale to cover the experimental temperature range $0<$ $T_{c}-T \leq 15000 \mathrm{~m}^{\circ} \mathrm{C}$ (here with $T_{1 \varphi}>T_{C}=45573 \pm$ $\left.1 \mathrm{~m}^{\circ} \mathrm{C}\right)$.

\section{Cell imaging and Image processing}

\subsection{Cell imaging of the meniscus position.}

The liquid-gas meniscus is observed from optical transmission imaging through the cell, using LED illumination and cell view observation with a CCD camera $(1024 \times 1024$ pixels). The pixel size corresponds to $12 \mu \mathrm{m}$ in the wide field-of-view imaging, well controlled from the two engraved circles on the external surface of each sapphire window. Additional small field-of-view (microscopy) imaging with $1.0 \mu \mathrm{m}$-pixel resolution of a typical object area $1 \times 1 \mathrm{~mm}^{2}$ in the central part of the fluid sample are also performed but not reported here. The cell images are also made by tuning the focal plane between the two window internal surfaces to control small optical perturbative effects related to any nonlinear light bending situations, e.g., due to a nonparallelism between tilted windows, wetting layerlensing effects, compressibility effects, or displacement of the optical axis of imaging lenses versus the exact center axis of the cylindrical fluid cell volume.

\subsection{Image processing of the cell position.}

Before the determination of the meniscus position, the image processing needs the exact pixel coordinates of the viewed fluid cell volume to be determined inside the images recorded for each $\llbracket i, X \rrbracket$ configuration.

The picture given in Fig. 3 for the $\llbracket 1, V \rrbracket$ configuration at $T=45473 \mathrm{~m}^{\circ} \mathrm{C}$ is chosen to briefly summarize the method that uses the line profile analysis provided by the NI Vision Assistant 2012 software. Each pixel point is characterized by its $\mathrm{x}$ (horizontal)-y (vertical) raw coordinates where the axis origin takes place on the top-left corner of the picture. Therefore, the line profiles provide the $x-y$ coordinates of the selected borderline points between the fluid and body cell (see A, B, C, T, \& R points in Fig. 3). The resulting position of the cell borderline (here a quasi-circle of $\sim 10.380-10.464 \mathrm{~mm}$ diameter, i.e., $~ 865-872$ pixels) can be controlled by the comparison with the position of the two engraved circles $(10 \pm 0.01 \mathrm{~mm}$ or $833.3 / 833.4$ pixels of diameter) on the external surface of the input and output windows. As an essential result, the (horizontal and vertical) pixel coordinates of the apparent center point $\mathrm{O}$ are the intrinsic characteristics parameters of the fluid volume position whatever each cell picture. The resulting estimation of the maximum error on the absolute position of any characteristic point of each profile line of each picture is \pm 0.5 pixels.

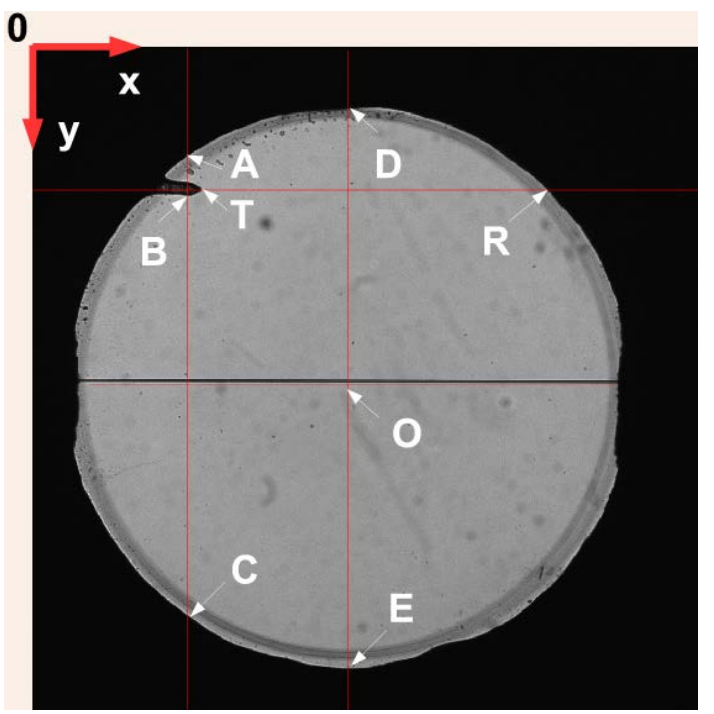

Fig. 3. Video picture of the ALIR5 cell for the $\llbracket 1, V \rrbracket$ configuration at temperature $T=45473 \mathrm{~m}^{\circ} \mathrm{C}$. Selected borderline points A, B, C, T, \& R between the fluid and cell body are used in the image processing to define the fluid sample cell position (especially the apparent center point O) inside the picture. Vertical line DE is used to analyse the meniscus position and shape as functions of temperature.

The next step compares similar line profiles obtained at different temperatures to probe the absence of thermal effect at the pixel level during the temperature timeline on each facility configuration.

The last step optimizes the matching of the selected characteristic points for two reversed similar configuration ( $1, V \rrbracket$ and $\llbracket 2, V \rrbracket$ for the chosen case). Indeed, the changes of the facility positions under the Earth's gravitational acceleration field induce small mechanical relative displacements due to the intrinsic clearance between the different (optical and mechanical) components. The present concern involves the cell (housed in the insert) in front of the video camera (located in the optical box of the facility). Therefore, this cell image matching step leads to the determination of the (horizontal and vertical) pixel shifts ( $\sim 2$ to 6 pixels, typically) between two reversed images of the viewed fluid cell volume.

\subsection{Image processing of the meniscus position.}

For each $\llbracket i, X \rrbracket$ case, the line profile analyses are then applied to the horizontal (or vertical) lines that are closer to the related $\mathrm{O}$ point (see for example the line DE in Fig. 3). The details of these analyses are not reported here, and only are illustrated in Fig. 4 the main results of these line profile analyses (for $\llbracket 1, V \rrbracket$ and $\llbracket 2, V \rrbracket$ configurations). The line profiles along DE give access to the position and shape of the meniscus 
at each temperature. Taking then reference from the $\mathrm{x}-\mathrm{y}$ position of a characteristic point of the viewed cell volume (such as the point $\mathrm{B}$ in the selected case of Fig. 3), the bare pixel distance of the meniscus position can be estimated. The temperature dependences of these bare distances are reported in Fig. 4 which illustrates (i) the well-defined crossing of the meniscus at finite temperature distance from the transition temperature, and (ii) the well-defined position of the volumetric median plane of the fluid cell.

Additional important results are also obtained, as the amplitude and shape of the capillary rising effect, the symmetrical matching of the meniscus position by fine tuning ( \pm 0.1 pixel, typically) of the apparent median plane for the two (slightly shifted) apparent cells, and the resulting noticeable symmetrical behavior of the capillary rising on the complete temperature range.

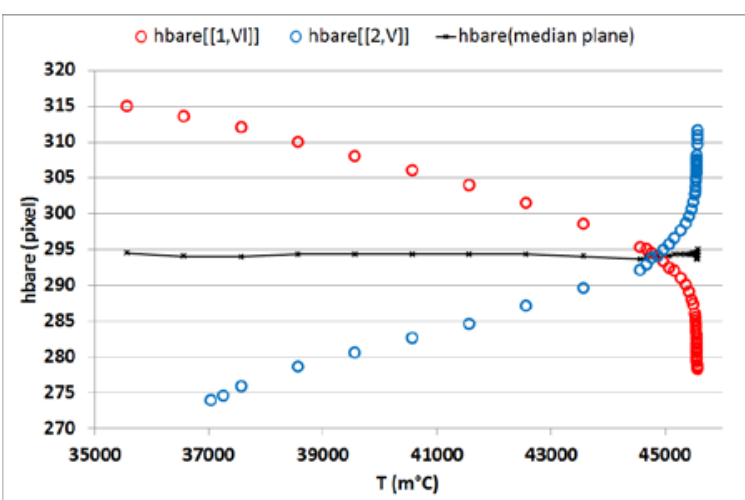

Fig. 4. Bare pixel distances of the meniscus as functions of the temperature for the $\llbracket 1, V \rrbracket$ (open red circles) and $\llbracket 2, V \rrbracket$ (open blue circles) configurations. Related bare pixel distance of the volumetric median plane of the cell. From reference to the y pixel coordinate of the B point in Fig. 3.

Finally, the essential feature of the image analyses reflects the combination of the highly symmetrical cell design, the small off-density criticality of the cell filling and the wide field-of-view cell imaging. Such a combination leads to estimate the absolute position of the volumetric median plane of the cell and the meniscus position with the \pm 0.5 pixel (i.e., $\pm 6 \mu \mathrm{m}$ ) resolution. Such a resolution is obtained whatever the $\llbracket i, X \rrbracket$ configurations, thanks to the similarity of the meniscus behavior and temperature crossing for two reverse positions under the gravity field acceleration. One noticeable remark concerns the (gas or liquid) filling of one half part of the dead volume (i.e., 7.0 $\mathrm{mm}^{3}$ ). Such a non-viewed fluid volume is thus similar to a viewed fluid median layer of $\langle\delta h\rangle_{f b}=$ $(1 / 2) V_{f b} / A_{f v} \simeq 263 \mu \mathrm{m}$ thickness (i.e., $\simeq 21.91$ pixels). The possible non-symmetrical effects related to the phase behavior in each windowless fluid volume can then easily be detected from the related viewed change of the meniscus position (see $\S 4$ ), while its minimum \pm 0.5 pixel variation only corresponds to $\pm 0.0675 \%$ of the total fluid volume, conform to the density precision requirements.

\section{Results}

Figure 4 shows that the pixel coordinate of the symmetrized meniscus positions, i.e., one half part noted $h_{i, X}$ of the differences between the related bare pixel distances, can be estimated from reference to the volumetric median plane of the cell in the selected configurations. The temperature behaviors of $h_{i, X}$ are reported in Fig. 5 for the eight $\llbracket i, X \rrbracket$ configurations. Except for both $\llbracket i, T \rrbracket$ and $\llbracket i, H \rrbracket$ cases (see below), the temperature crossing of the volumetric median plane of the cell occurs in the range $44673 \leq$ $T_{\text {cross }}\left(\mathrm{m}^{\circ} \mathrm{C}\right) \leq 44973 \quad$ (i.e., $\quad T=T_{c}-\{600 ; 900\}$ $\mathrm{m}^{\circ} \mathrm{C}$ (accounting for \pm 0.5 pixel $\sim\left|\delta h_{i, X}\right| \leq \pm 6 \mu \mathrm{m}$ uncertainty).

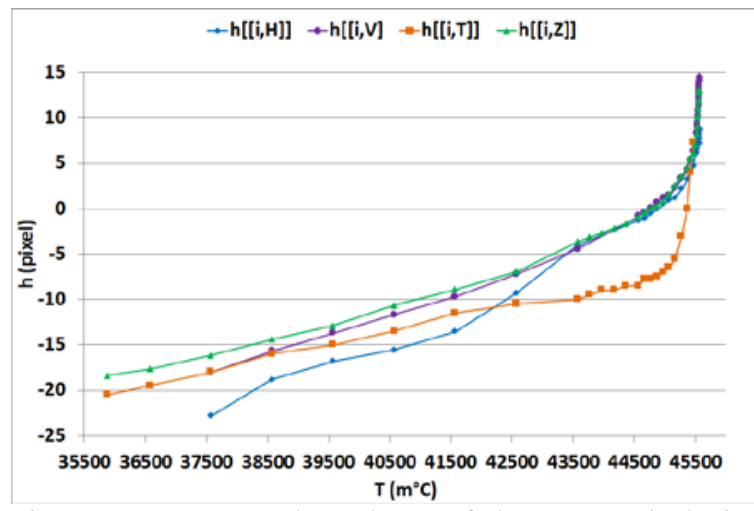

Fig. 5. Temperature dependence of the symmetrical pixel shift of the meniscus position from reference to the corresponding volumetric median plane, for the eight $\llbracket i, X \rrbracket$ configurations. 1 pixel $=12 \mu \mathrm{m}$.

The meniscus behaviour for $\llbracket i, T \rrbracket$ cases is clearly affected by a significant non-symmetrical effect of wetting liquid phase inside the dead cell volume. Indeed that is the only configuration where the expected gas-like dead volume appears on a position which can easily be connected to the liquid side of the cell by the capillary effects. Accounting for the above remark about $\langle\delta h\rangle_{f b}$, it seems that $1 / 3$ of this gas-like dead volume can be filled by liquid around $T_{\text {cross }}$. Obviously, this over liquid trapping decreases with temperature since the meniscus position is more and more lowered below the corresponding fill-in channel.

Such plausible non-symmetrical liquid wetting effects can also occur for $\llbracket i, H \rrbracket$ cases, particularly observed at low temperatures $\left(T \simeq 43573 \mathrm{~m}^{\circ} \mathrm{C}\right.$ ) where capillary condensation can exist in the small fill-in channel. Conversely only a very small part $(2 / 10)$ of the dead volumes seems to be responsible of the $h_{i, H}$ differences compared to the $\llbracket i, V \rrbracket$ or the $\llbracket i, Z \rrbracket$ configuration cases.

\section{Modeling}


The following modeling starts from the initial result given in Ref. [22] for an ideal constant cylindrical volume of the fluid sample with radius $R$ filled at a small liquid-like off-critical density $\langle\delta \tilde{\rho}\rangle\rangle$ 0 . The horizontal position $h \ll R$ of the liquid-gas meniscus from reference to the horizontal cell median plane is written as follows

$$
\frac{h}{R}=\frac{\pi}{4} \frac{\langle\delta \widetilde{\rho}\rangle-\Delta \widetilde{\rho}_{d}}{(\Delta \widetilde{\rho})_{L V}}
$$

where

$$
\begin{aligned}
& \Delta \tilde{\rho}_{d}=\frac{\rho_{L}+\rho_{V}}{2 \rho_{c}}-1 \\
& (\Delta \tilde{\rho})_{L V}=\frac{\rho_{L}-\rho_{V}}{2 \rho_{c}}
\end{aligned}
$$

$\rho_{L}$ and $\rho_{V}$ are the coexisting liquid and vapor densities at temperature $T<T_{c}$, respectively. In this ideal cylindrical cell, the fluid compressibility and capillary effects are neglected, while only simple geometrical considerations are used to define the liquid-vapor distribution which results from the fluid mass conservation at any $T$.

For the present ALIR5 case, the additional $\Gamma$-like symmetrical windowless fluid volume is accounted for rewriting the total volume $V_{f}$ as $V_{f}=$ $\pi R^{2} e_{f}(1+x)$, with $x=V_{f b} / V_{f v} \simeq 0.063$. As a direct consequence, the fluid (gas or liquid) filling of the half part of the dead volume is measured by the ratio $\langle\delta h\rangle_{f b} / R= \pm \pi x / 4 \simeq \pm 0.0496 .\langle\delta h\rangle_{f b}$ is the above viewed change of the meniscus position around the fluid median plane (i.e., $\simeq \pm 21.91$ pixels or $\simeq \pm 263$ $\mu \mathrm{m})$.

The thermal effects are accounted for by exchanging the $\langle\delta \tilde{\rho}\rangle$ term of Eq. (1) by

$$
\langle\delta \tilde{\rho}\rangle_{T}=\langle\delta \tilde{\rho}\rangle_{T_{C}}\left(1+3 \alpha_{T} T_{c} \Delta \tau^{*}\right)+3 \alpha_{T} T_{c} \Delta \tau^{*}
$$

The above temperature dependence of $\langle\delta \tilde{\rho}\rangle_{T}$ is obtained from a linear change of the cell mean density written as $\rho_{T}=\langle\rho\rangle_{T_{C}}\left(1+3 \alpha_{T} T_{c} \Delta \tau^{*}\right) . \alpha_{T}=1.8 \times$ $10^{-6} \mathrm{~K}^{-1}$ is the thermal dilatation coefficient of the $\mathrm{CuCo}_{2} \mathrm{Be}$ alloy. In the temperature range $T_{c}-T \leq 2$ $\mathrm{K}$, the cell thermal dilatation effect is lower than $5.5 \%$. These effects reach $29 \%$ at $T_{c}-T \simeq 10 \mathrm{~K}$. Such effects need to be accounted for in the cell filling process at the laboratory temperature (they are $\simeq 60 \%$ in the temperature range at $T_{\text {lab }} \simeq 20-25^{\circ} \mathrm{C}$.

The liquid wettability effects are estimated as the form of an equivalent height down $\langle\delta h\rangle_{c a}$ of the meniscus position

$$
\langle\delta h\rangle_{c a} \propto l_{c a}^{2}\left(1-\frac{\pi}{4}\right) \frac{2 R+e_{f}}{R e_{f}}
$$

$\langle\delta h\rangle_{c a}$ corresponds to the thickness of a horizontal liquid planar layer having similar volume to the total wetted liquid volume on sapphire and cell body. To derive Eq. (5), it is assumed an ellipsoidal shape for the meniscus capillary rising such that the product of characteristic size parameters are proportional to the squared capillary length $l_{c a}^{2}$ (so-called the capillary constant $a^{2}$ ) i.e., $l_{c a}^{2}=l_{0}^{2}\left|\Delta \tau^{*}\right|^{2 v-\beta}$, with $2 v-\beta=$ 0.935 and asymptotic amplitude $l_{0}^{2} \simeq 3.84 \mathrm{~mm}^{2}$ [26]. The $l_{c a}^{2}$ behavior compares well with the effective singular behavior $a^{2}=\left(3.94 \mathrm{~mm}^{2}\right)\left|\Delta \tau^{*}\right|^{0.944}$ [27][28]. The validity of $l_{c a}^{2} \propto a^{2}$ is controlled from the apparent size of the meniscus thickness due to the meniscus capillary rising. Finally only the proportional amplitude (of value 1.44, see $\S 6$ ) of Eq. (5) remains as the adjustable parameter at large temperature distances from $T_{c}$. However, in the temperature range $T_{c}-T \leq 3 \mathrm{~K}$, it is noticeable that $\langle\delta h\rangle_{c a}$ remains lower than one half pixel $(<6 \mu \mathrm{m})$, i.e., $\langle\delta h\rangle_{c a} / R<1.2 \times 10^{-3}$. In such a case, it is also important to note the large value of the ratio $\langle\delta h\rangle_{f b} /\langle\delta h\rangle_{c a} \simeq 50$.

The final functional form of $h$ then writes as follows

$$
\frac{h}{R}=\frac{\pi}{4} \frac{\langle\delta \widetilde{\rho}\rangle_{T}-\Delta \widetilde{\rho}_{d}}{(\Delta \widetilde{\rho})_{L V}}(1+x)-\frac{\langle\delta h\rangle_{c a}}{R}
$$

where only the fluid compressibility effects still remain neglected. These latter effects can be observed from the grid deformation and the related local turbidity on the both sides of the vapor-liquid meniscus and are only noticeable in the temperature range $T_{c}-T \leq 5 \mathrm{~m}^{\circ} \mathrm{C}$.

\section{Discussion}

When the capillary rise effects are negligible (i.e. in the temperature range $T_{c}-T \leq 3 \mathrm{~K}$ ), Eq. (6) shows that the meniscus behavior in a cell with finite positive value of $\langle\delta \tilde{\rho}\rangle_{T}$ crosses the cell median plane at a single temperature $T_{\text {cross }}$ where $h=0$, i.e., $\langle\delta \tilde{\rho}\rangle_{T_{\text {cross }}}=\Delta \tilde{\rho}_{d}$. A first approach considers the linear functional form of $\tilde{\rho}_{d}$ such as

$$
\tilde{\rho}_{d}=1+a_{d}\left|\Delta \tau^{*}\right|
$$

where the value $a_{d}=0.84 \pm 0.015$ of the slope of rectilinear diameter results from the coexisting density data on the complete two-phase range. The above central estimation of $T_{\text {cross }}=44823 \mathrm{~m}^{\circ} \mathrm{C}$ then leads to $\langle\delta \tilde{\rho}\rangle_{T_{\text {cross }}} \cong\langle\delta \tilde{\rho}\rangle_{T_{c}}=0.20 \% \quad$. Earth's-based visualization of the meniscus behavior in the ALIR5 cell is well an academic benchmark experiment where the resolution in the image processing at the pixel level is of prime interest for accurate determination of the mean filling density of the fluid cell. This experiment authorizes a preliminary checking (without accounting for the compressibility effects) of the validity of the expected singular top shape of the coexisting density curve and its related singular 
diameter presumably satisfying the different theoretical functional forms issued from the literature.

The singular top-shape of the coexistence curve $(\Delta \tilde{\rho})_{L V}\left(\left|\Delta \tau^{*}\right|\right)$ for $\left|\Delta \tau^{*}\right| \leq 10^{-2}$ can be predicted without adjustable parameter [4] from the theoretical master crossover functions estimated from the massive renormalization scheme. Nevertheless, any other effective power laws to describe $(\Delta \tilde{\rho})_{L V}\left(\left|\Delta \tau^{*}\right|\right)$ of $\mathrm{SF}_{6}$ (such as for instance $(\Delta \tilde{\rho})_{L V}=$ $1.7147\left|\Delta \tau^{*}\right|^{0.3271}+0.8203\left|\Delta \tau^{*}\right|^{0.8215}-$

$1.4396\left|\Delta \tau^{*}\right|^{1.2989}$ from Ref. [20]) do not modify the following analysis, especially considering the temperature range $0.03 \leq T_{c}-T<3 \mathrm{~K}$ where $\left|\delta h_{i, X}\right| \leq 100 \mu \mathrm{m}$ (i.e., $\left|\delta h_{i, X}\right| \leq 8$ pixels.

The second approach thus introduces the singular functional forms of $\Delta \tilde{\rho}_{d}$ as follows

$$
\Delta \tilde{\rho}_{d}=\frac{A_{\beta}\left|\Delta \tau^{*}\right|^{2 \beta}+A_{\alpha}\left|\Delta \tau^{*}\right|^{1-\alpha}+A_{1} \Delta \tau^{*}+A_{\Delta}\left|\Delta \tau^{*}\right|^{x_{\Delta}}}{1+a_{\Delta}\left|\Delta \tau^{*}\right|^{\Delta}}
$$

Equation (8) results from the various complete field mixing (CFM) models predicting the singular asymmetry with adjustable amplitudes. The amplitude sets obtained from the Weiner's data fitting [10] are given in Table 1 with $\alpha=0.109, \beta=0.326, \Delta=$ 0.52 , and $x_{\Delta}=1-\alpha+\Delta$. Any adjustment of (at least three) free amplitudes appears Weiner-like compatible whatever the used additive forms of the power laws and exponents involved in Eq. (8).

Table 1. $\mathrm{SF}_{6}$ parameters for Eq. (8)

\begin{tabular}{lllll}
\hline & {$[7]$} & {$[7]$} & {$[7]$} & {$[9]$} \\
\hline$A_{\beta}$ & 0 & 1.124 & 1.0864 & 0.46028392 \\
$A_{\alpha}$ & 6.365 & -9.042 & -7.990 & -0.6778981 \\
$A_{1}$ & -10.13 & 11.37 & 9.770 & 0.13516245 \\
$A_{\Delta}$ & 8.080 & -3.354 & 0 & 0 \\
$a_{\Delta}$ & 0 & 0 & 3.318 & 0 \\
\hline
\end{tabular}

The corresponding estimations of $(h / R)$ as a function of $T_{c}-T$ are illustrated in Fig. (6) where the value $\langle\delta \tilde{\rho}\rangle_{T_{C}}=0.20 \%$ is fixed. Only the experimental results for the $\llbracket i, V \rrbracket$ (full blue circles) and $\llbracket i, Z \rrbracket$ (full green triangles) configurations are used. For the rectilinear diameter case, the dotted and full blue curves are for the use of Eqs. (6)-(7), without or with capillary correction term, where are introduced the corresponding predictions of Ref. [4] (see above). For the critical hook case, the brown, green, and pink full curves are for the use of Eqs. (6) and (8) with parameters of columns 2,3 , and 5 (without visible difference between the green curve (column 3 case) and green circles (column 4 case). In addition, published $\rho_{L} / \rho_{c}$ and $\rho_{V} / \rho_{c}$ Weiner's data (Table X of [19]), earlier supporting the diameter deviation on $\mathrm{SF}_{6}$, can directly be used to estimate the various terms of Eq. (6). In Fig. 6, the orange full diamonds are for the corresponding meniscus positions at the Weiner's experimental $T_{c}-T$ values.

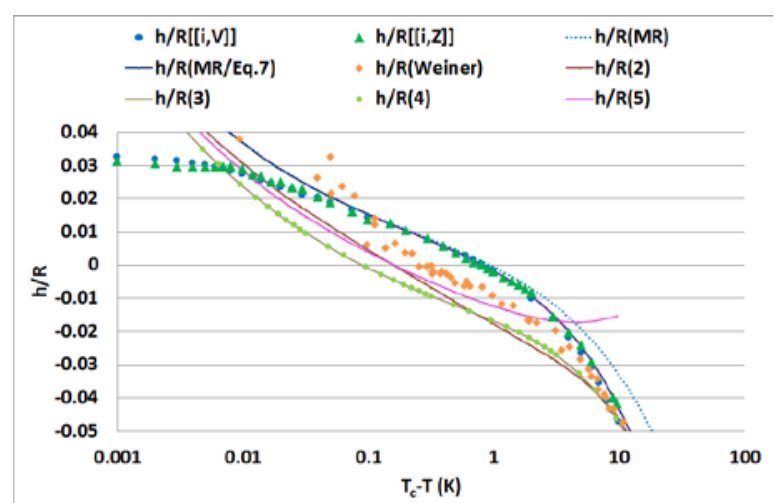

Fig. 6. Comparison between experimental and modelling results of $(h / R)$ as functions of $T_{c}-T$, fixing $\langle\delta \tilde{\rho}\rangle_{T_{c}}=$ $0.20 \%$.

Clearly, only the $(h / R)$ calculations for the linear density diameter case are in good agreement with the experimental data, especially in the two temperature decades $25 \leq T_{c}-T<2500 \mathrm{mK}$ of prime interest regarding the neglected effects. In addition to an intrinsic questioning of the Weiner's measurements of the $\mathrm{SF}_{6}$ diameter deviation [28], the noticeable inconsistency observed in Fig. 6 can be attributed to a non-realistic estimation of the uncertainty on $\left(\rho_{L} / \rho_{c}\right)+\left(\rho_{V} / \rho_{c}\right)$ Weiner values (at least onedecade larger than the maximum amplitude $(0.5 \%)$ of the hook-like deviation), especially close to critical temperature (see Fig. 1). More generally, the systematic large data dispersion on the complete temperature range of the Weiner's data mainly seems due to the Weiner's values of the critical parameters $\rho_{c}$ (density), $\varepsilon_{c}$ (dielectric constant), and then $C M_{c}=$ $\left(1 / \rho_{c}\right)\left(\varepsilon_{c}+1\right) /\left(\varepsilon_{c}+2\right) \quad$ (Clausius-Mossotti constant), which are significantly different ( $-1.5 \%$, $10.9 \%$, and $-3.3 \%$, respectively) from the literature ones [29].

\section{Conclusions}

The $(h / R)$ modeling from Eqs. (6) and (7) is comparable (in amplitude and uncertainty) with the Earth's-based measurements. Along the off-critical thermodynamic path of $\langle\delta \tilde{\rho}\rangle=+0.20_{-0.04}^{+0.04} \%$, the careful imaging analysis of the $\mathrm{SF}_{6}$ two-phase domain appears well understood without the supplementary addition of any singular hook-shaped deviation in the rectilinear density diameter. The main part of the uncertainty in the rectilinear density diameter remains due to the actual level of precision $(0.21 \%)$ for the $\mathrm{SF}_{6}$ critical density value. In such an uncertainty range, the cell thermal dilatation, the fluid compressibility, the fluid coexisting densities and the liquid wettability effects can be well-controlled from the highly symmetrical ALIR5 cell design. The slope of the $\mathrm{SF}_{6}$ linear density diameter seems the only remaining adjustable parameter, leading to questionable applicability of the complete field mixing to the simple fluid case. 
Future modeling approaches [30] will be focused on the estimation of the fluid compressibility effects using an upgraded version of the universal parametric equation of state. Moreover ongoing experimental works will be performed to account for the eventual contribution of the $\mathrm{SF}_{6}$ purity on the critical parameters before to close in a sure manner the debating situation about the density diameter behavior close to its critical point.

\section{Acknowledgements}

We thank all the CNES, CNES-CADMOS, NASA, and associated industrial teams involved in the DECLIC facility project. CL, SM, YG, DB, are grateful to CNES for the financial support. They are also grateful to Philippe Bioulez and Hervé Burger for their operational support at CADMOS. The research of I.H. was carried out at Jet Propulsion Laboratory, California Institute of Technology, under a contract with NASA.

\section{References}

[1] K. G. Wilson, The renormalization group: Critical phenomena and the Kondo problem, Rev. Mod. Phys. 47, 773 (1975).

[2] J. Zinn-Justin, Quantum Field Theory and Critical Phenomena, 4th ed. (Clarendon, Oxford, 2002).

[3] M. E. Fisher, Correlation Functions and the Critical Region of Simple Fluids, J. Math. Phys. 5, 944 (1964).

[4] Y. Garrabos, C. Lecoutre, S. Marre, D. Beysens, and I. Hahn, Crossover equation of state models applied to the critical behavior of xenon, J. Stat. Phys. 158, 1379-1412 (2015).

[5] M. Barmatz, I. Hahn, J.A. Lipa, R.V. Duncan, Critical phenomena in microgravity: Past, present, and future, Rev. Mod. Phys. 79,1 (2007).

[6] L. Cailletet et E. Mathias, Recherches sur les densités des gaz liquéfiés et de leurs vapeurs saturées, J. Phys. Theor. Appl., 5, 549-564 (1886).

[7] R. R. Singh and K. S. Pitzer, Rectilinear diameters and extended corresponding states theory, J. Chem. Physics 92, 3096-3099 (1990).

[8] M. E. Fisher and G. Orkoulas, The YangYang anomaly in fluid criticality: experiment and scaling theory, Phys. Rev. Lett. 85, 696-699 (2000).

[9] Y. C. Kim, M. E. Fisher and G. Orkoulas, Asymmetric fluid criticality. I. Scaling with pressure mixing, Phys. Rev. E 67, 061506 (2003).

[10] Y. C. Kim and M. E. Fisher, Singular coexistence-curve diameters: Experiments and simulations, Chem. Phys. Letters 414, 185-192 (2005).

[11] M. A. Anisimov and J. Wang, Nature of asymmetry in fluid criticality, Phys. Rev. Lett. 97, 025703 (2006).
[12] J. Wang and M. A. Anisimov, Nature of vapor-liquid asymmetry in fluid criticality, Phys. Rev. E 75, 051107 (2007).

[13] C. A. Cerdeirina, G. Orkoulas, and M. E. Fisher, Soluble model fluids with complete scaling and Yang-Yang features, Phys. Rev. Lett. 116, 040601 (2016).

[14] F. J. Wegner, Corrections to scaling laws, Phys. Rev. B 5, 4529 (1972).

[15] C. Bagnuls and C. Bervillier, Classical-tocritical crossovers from field theory, Phys. Rev. E 65, 066132 (2002).

[16] Y. Garrabos and C. Bervillier, Master crossover functions for one-component fluids, Phys. Rev. E 74, 021113 (2006).

[17] C. Lecoutre, R. Guillaument, S. Marre, Y. Garrabos, D. Beysens, and I. Hahn, Weightless experiments to probe universality of fluid critical behavior, Phys. Rev. E 91, 060101(R) (2015).

[18] J. Weiner, K. H. Langley, and N. C. Ford Jr., Experimental evidence for a departure from the law of the rectilinear diameter, Phys. Rev. Lett. 32, 879 (1974).

[19] J. Weiner, Breakdown of the law of rectilinear diameter, Ph.D. thesis, University of Massachusetts, Amherst (1974).

[20] M. Ley-Koo and M. S. Green, Revised and extended scaling for coexisting densities of $\mathrm{SF}_{6}$, Phys. Rev. A 16, 2483-2487 (1977).

[21] ALIR5 test cell was selected among a series of 10 identical ALIR $n$ cell (with $n=1$ to 10 ). The series have provided statistical evaluation of the fluid volume and fluid mass uncertainties $(0.05 \%$ and $0.1 \%$, respectively), related to the similar procedures used from a single alloy ingot / machining / mounting / filling steps and the selected fluid purity (fixing $\rho_{c}=$ $\left.0.742 \mathrm{gcm}^{-3}\right)$.

[22] C. Morteau, M. Salzmann, Y. Garrabos, and D. Beysens, in Proceedings of 2nd European Symposium Fluids in Space, Edited by A. Viviani (Edizioni Jean Gilder Congressi srl., Naples, 1996), pp. 327-333.

[23] B. Zappoli, D. Beysens, and Y. Garrabos, Heat Transfers and Related Effects in Supercritical Fluids (Springer, Amsterdam, 2015), pp. 199-211, and references therein.

[24] DECLIC-CNES,

http://smsc.cnes.fr/DECLIC/index.htm ， 2018 (accessed 19 February 2018)

[25] For ALIR5, $\langle\delta \tilde{\rho}\rangle_{T_{c}}=0.20 \%$ and $(\Delta \tilde{\rho})_{L V}=$ $B\left|\Delta \tau^{*}\right|^{\beta}$, then $T_{c}-T_{\text {coex }} \simeq 0.4 \mu \mathrm{K}$, with $\mathrm{B}=1.596, \beta$ $=0.32575$ and $T_{c}$ (pure $\mathrm{SF}_{6}$ ) $=318.733 \mathrm{~K}$.

[26] Y. Garrabos, F. Palencia, C. Lecoutre, D. Broseta, B. LeNeindre, and C. Erkey, Master singular behavior for the Sugden factor of one-component fluids near their gas-liquid critical point, Phys.Rev. E 75, 061112 (2007).

[27] W. Rathjen and J. Straub, in Proceedings of 7th Symposium on Thermophysical Properties, Ed. A. Cezairliyan (ASME, New York, 1977), pp. 839-850. 
[28] M. R. Moldover and R. W. Gammon, Capillary rise, wetting layers, and critical phenomena in confined geometry, J. Chem. Phys. 80, 528-535 (1984).

[29] Weiner's values are $\rho_{c}=(0.731 \pm 0.001)$ g.cm ${ }^{-3}, \quad \varepsilon_{c}=0.262 \pm 0.010$, and $C M_{c}=$ $(0.1605 \pm 0.0010) \quad \mathrm{cm}^{3}$.mole ${ }^{-1}$.The most accurate literature values are $\rho_{c}=(0.742 \pm 0.0015) \mathrm{gcm}^{-3}$, $\varepsilon_{c}=0.2758 \pm 0.0018 \quad$, and $C M_{c}=(0.1657 \pm$ $0.0007) \mathrm{cm}^{3}$.mole ${ }^{-1}$.

[30] Y. Garrabos, C. Lecoutre, S. Marre, D. Beysens, and I. Hahn, Liquid-vapor rectilinear diameter revisited, Phys. Rev. E 97, 020101(R) (2018). 
